\title{
Two Decades of Cancer Genetics: From Specificity to Pleiotropic Networks
}

\author{
S. GRISENDi AND P.P. PANDOLFI \\ Cancer Biology \& Genetics Program, Department of Pathology, Sloan-Kettering Institute, \\ Memorial Sloan-Kettering Cancer Center, New York, New York 10021
}

\begin{abstract}
Modeling cancer in mice has reached an even greater relevance in the field of hematological malignancies, due to the already advanced characterization of the molecular basis of many hematological disorders. These mouse models have often allowed us to achieve insight into the pathogenesis of the human disease as well as to test novel therapeutic modalities in preclinical studies. However, one of the most rewarding cultural shifts triggered by these modeling efforts stems from what was originally perceived as background noise or modeling inaccuracy. Manipulation of the involved genes often triggered cancer susceptibility in cell types other than the hematopoietic lineages. This prompted us to challenge a fundamental misconception in cancer genetics that the approximately 200 genes directly involved in chromosomal translocations associated with hematopoietic malignancies are specifically and functionally restricted to leukemia/lymphoma pathogenesis only. The genetics underlying the pathogenesis of leukemia and lymphoma have historically been regarded as distinct from those underlying the pathogenesis of solid tumors because hematopoietic malignancies are often associated with characteristic chromosomal translocations that are leukemia- or lymphoma-specific. In this paper, we discuss how leukemia/lymphoma genes indeed participate in fundamental proto-oncogenic and growth-suppressive networks and may play a wider role in cancer pathogenesis. We focus on paradigmatic examples such as c-myc and PML, as well as on more recent findings from our laboratory concerning the role of NPM in tumorigenesis.
\end{abstract}

In this review paper, we discuss how recent findings in our laboratory have allowed us to challenge a self-inflicted dogma in cancer genetics whereby the genes involved in the pathogenesis of hematopoietic malignancies would be "specific" to these disorders. Although this belief is clearly wrong, as is probably obvious to the majority of the people reading this paper, it still surprisingly permeates the imagination of a vast number of people involved in cancer research and, importantly, in the clinical practice of cancer management and therapy. This misconception has in turn led to a dangerous conceptual drift and important implications both investigational and practical. First of all, very few laboratories are actively studying the possible direct involvement of leukemia/lymphoma genes in the pathogenesis of non-hematopoietic cancers; second, on the basis of this tenet, the motivation of pharmaceutical companies to embark on drug discovery efforts for "leukemia/lymphoma genes" is often low, as many hematopoietic malignancies are extremely rare, therefore not constituting a sufficient justification for a business enterprise.

As mentioned above, the "dogma" is self-inflicted, and it is in fact the outcome of 20 years, or more, of dramatic progress in elucidating the molecular genetics underlying hematopoietic malignancies. This has led to a novel molecular reclassification of these malignancies on the basis of distinctive genetic lesions (often chromosomal translocations either leading to the generation of fusion genes as frequently observed in myelogenous leukemia, or to the deregulated expression of proto-oncogenes as is many times the case in lymphomas) (Fig. 1). Although these advances have certainly had a tremendous impact on our ability to better diagnose these diseases and to tailor therapeutic intervention modalities, the enthusiasm that ensued led to the misleading notion that the genes involved in these translocations are "specifically" involved in the pathogenesis of hematopoietic malignancies, even though their expression pattern is often very broad and dynamic. The notion of specificity has been further exacerbated by two facts: (1) Chromosomal translocations are thought to be extremely rare in non-hematopoietic tumors with few notable exceptions, e.g., Ewing's sarcoma (possibly another key misconception, as a very recent study describing the identification of recurrent chromosomal translocations in a very high percentage of prostate cancers strongly suggests [Tomlins et al. 2005]); (2) hematopoietic-associated chromosomal translocations are specifically associated with distinct subtypes of leukemia and lymphoma (e.g., chromosomal translocations involving the $R A R \alpha$ gene specifically associated with acute promyelocytic leukemia [APL], as we discuss in this review). However, the caveat is, as it clearly turns out when analyzing the published literature, that in very few instances these genes have been thoroughly analyzed in non-hematopoietic neoplasms, and a systematic analysis has never been undertaken, despite numerous high-throughput searches for cancer genes involved in solid tumor pathogenesis.

In our laboratory, we have decided to undertake a systematic study to learn whether leukemia/lymphoma genes are indeed implicated in solid tumor pathogenesis. This "inside out" cultural shift is proving extremely rewarding and has already led to unexpected and exciting outcomes that we discuss here.

\section{BREAKING THE DOGMA AT THE ONSET: c-myc AS A GENERAL PROTO-ONCOGENE}

A compelling example of the broad function of genes first identified as associated with hematological malignan- 

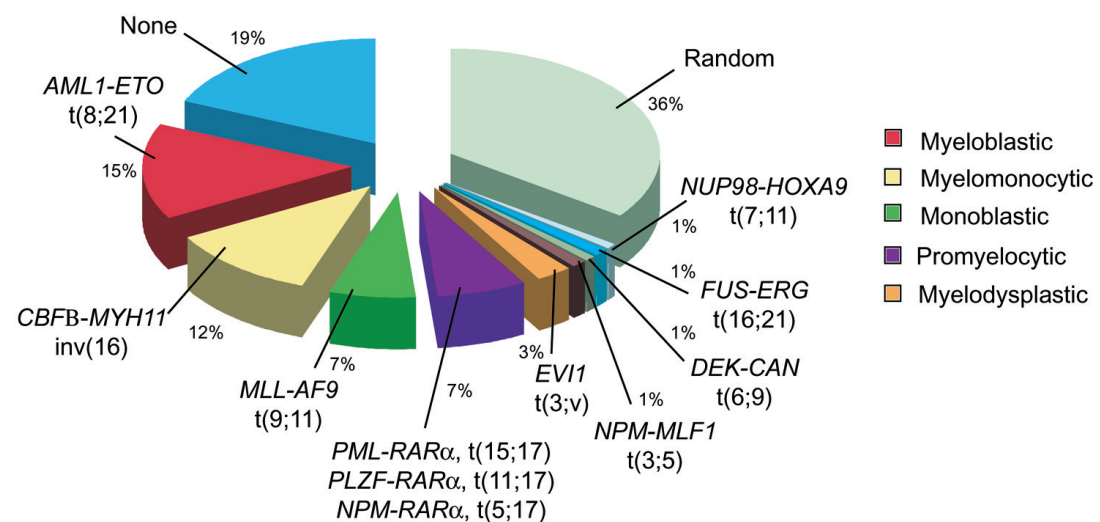

Figure 1. Aberrant transcription in AML. Mostly recurrent chromosomal translocations associated with acute myeloid leukemia (AML) and their relative distribution (adapted from T. Look). Such structural abnormalities represent cytological and molecular markers for the diagnosis and prognosis of the disease. In many cases, the genetic basis of the disease has been firmly established, and many translocations and mutations are recognized as critical in the leukemia pathogenesis. In the majority of cases, genetic alterations in AML affect transcription factors that have an important role in normal hematopoiesis, as in the case of the retinoic acid receptor gene $(R A R \alpha)$, which translocates to several different partner genes (PML, PLZF, NPM1, etc.) in acute promyelocytic leukemia (APL).

cies is represented by the proto-oncogene c-myc. This powerful oncogene was one of the first proto-oncogenes to be identified as a mammalian homolog of the viral transforming oncogene, $\mathrm{v}-m y c$, responsible for avian myelocytomatosis, and soon recognized as a crucial causative agent in the pathogenesis of human lymphoma, where it is activated through chromosomal translocations (DallaFavera et al. 1982; Vennstrom et al. 1982). Virtually every case of Burkitt's lymphoma (BL) involves the juxtaposition of c-myc, located on chromosome 8, with regulatory elements of the immunoglobulin $\mu$ heavy chain or $\lambda$ and $\kappa$ light chains located on chromosome 14, 22, or 2, respectively (for review, see Spencer and Groudine 1991). Although the positions of the chromosomal breakpoints relative to the c-myc gene may vary considerably in individual cases of BL, the consequence of the translocations is invariably the same-deregulation of c-myc expression-leading to uncontrolled cellular proliferation.

c-myc was also one of the earliest oncogenes demonstrated to induce tumorigenesis in animal models: In one of the first and most popular transgenic models developed 20 years ago, it was shown in fact that transgenic c-myc under control of the immunoglobulin heavy-chain E $\mu$ enhancer predictably caused malignant B-cell tumors in mice (Adams et al. 1985). The E $\mu$-myc model had a large impact on the field of B-cell neoplasms and lymphomagenesis. This mouse model gave rise to countless studies concerning myc-dependent apoptosis, cooperative tumorigenesis allowing the identification of oncogenes and tumor suppressor genes that collaborate with c-myc in neoplastic development, the identification of cellular pathways that determine the susceptibility or resistance of myc-driven tumors to chemotherapeutic agents, and many other aspects of myc biology.

Over the last two decades, the understanding of the function of c-myc in normal cells and in cancer cells has significantly progressed. It is now clear that uncoupling of myc expression from cell cycle and cellular environmental controls is crucial for myc-mediated cellular transformation and subsequent tumorigenesis. Even more significantly, it is now clear that c-myc oncogenic activation occurs through different mechanisms and, importantly, that c-myc plays a direct role not only in a variety of hematological tumors but also in a large fraction of solid cancers (for review, see Oster et al. 2002). Numerous studies have established that the c-myc gene is in fact constitutively expressed or mutated in approximately $30 \%$ of human cancers (breast, prostate, colon, small-cell lung cancer, and melanoma; for review, see Nesbit et al. 1999). Whereas constitutive or deregulated expression of c-myc (which may be affected in cis by signaling pathways that converge at the myc promoter, or in trans by genomic rearrangements that juxtapose the gene to ectopic enhancers or gene amplification) results in elevated myc expression levels, mutations in the protein encoding region may diminish myc degradation in the ubiquitinproteasome pathway and therefore leads to stabilization and up-regulation of the oncoprotein.

All these observations have been accompanied by compelling evidence that c-myc plays a role in the pathogenesis of cancer at large from the use of mouse models. A multitude of transgenic models have been generated demonstrating that myc overexpression can induce tumorigenesis in most tissues (for review, see Morgenbesser and DePinho 1994). Importantly, recently generated conditional transgenic models have been used to demonstrate that even a brief inactivation of myc can reverse tumorigenesis (for review, see Arvanitis and Felsher 2005). Thus, the universal deregulation of c-myc expression in cancer makes it an attractive target for therapeutic intervention, and its important role in tumor maintenance suggests that its functional inactivation may constitute an effective treatment for many types of cancer.

\section{LEUKEMIA GENE SPECIFICITY: THE DOGMA STILL PERSISTS}

In the years following the cloning of the Burkitt's lymphoma-associated chromosomal translocation and the identification of c-myc, the idea that the molecular genet- 
ics of leukemia and lymphoma could be somewhat distinct from that of other tumors carved its way in the perception of many, and for compelling reasons. First of all, as mentioned above, leukemias and lymphomas are associated with specific chromosomal translocations that mediate activation of proto-oncogenes by their juxtaposition with promoter sequences (e.g., c-myc) or generate fusion of genes, whereas chromosomal translocations are rarely observed in solid tumors. Second, as both oncogene products and fusion proteins are often transcription factors or chimeric transcription factors, deregulated transcriptional control was thought to be the crucial and etiologically relevant force underlying hematopoietic malignancies, as opposed to solid tumors where aberrant signaling would be the most common underlying mechanism (once again an obvious misconception, as the examples of the $\mathrm{Rb}$ and p53 tumor suppressors clearly demonstrate). Third, the dramatic acceleration that our understanding of the molecular genetics of leukemia and lymphoma experienced in the 1990s (more than 200 genes have been directly implicated to date) has further corroborated the notion of the genetic uniqueness of hematopoietic malignancies. Although it is tremendously useful and conceptually rewarding to be able to diagnose one AML or lymphoma subtype almost in real time by utilizing convenient and affordable molecular tools such as RT-PCR, this perception of specificity slowly but surely insinuated that perhaps these genes of hematopoietic malignancies would not be perturbed in other tumor types. Although proto-oncogenic receptor tyrosine kinases and classic tumor suppressor genes (e.g., Rb, p53, PTEN) are also mutated/lost in hematopoietic malignancies, experimental evidence that the converse is true for the 200 or so leukemia/lymphoma genes is still lacking. To date, in only a few instances have alterations in AML-related genes been firmly associated with the pathogenesis of other tumor types (e.g., RUNX3, an AML1 family member, in gastric carcinomas [ $\mathrm{Li}$ et al. 2002] or $\mathrm{C} / \mathrm{EBP} \alpha$, which is down-regulated in a significant percentage of non-small-cell lung cancers [Halmos et al. 2002]).

We advocate here that the role of the vast majority of these leukemia/lymphoma genes in tumorigenesis at large has not yet been thoroughly investigated and that a systematic analysis is in fact warranted, discussing specific examples directly stemming from our own work.

\section{THE APL PARADIGM AND PROMYELOCYTIC LEUKEMIA}

Acute promyelocytic leukemia (APL), the M3 subtype in the FAB classification of AML, represents the perfect example of a form of leukemia where the molecular genetics currently informs the diagnosis, the prognosis, the management, and the treatment of the disease, and where the knowledge of the molecular basis had a direct and major impact on the development of targeted therapeutic modalities (Melnick and Licht 1999; Piazza et al. 2001). APL was considered invariably fatal only 20 years ago, whereas the current cure rate approximates $90 \%$, representing a genuine triumph of contemporary molecular medicine. Yet, these dramatic progresses have made leukemias such as APL or chronic myelogenous leukemia (CML) the archetypes on which the misconception of "hematopoietic specificity" rests: distinct AML subtype, specific molecular basis, specific treatment, and specific genes.

Indeed, the molecular genetics of APL is extremely specific to this AML subtype. APL is invariably associated with chromosomal translocations where the retinoic acid receptor $(R A R \alpha)$ gene fuses with variable partner genes (referred to as $\mathrm{X}$ genes/proteins). $R A R \alpha$ fuses to the $P M L$ gene in the vast majority of APL cases (>95\%), and in the remaining cases to the PLZF, NPMI, NuMA, and STAT5 genes (for review, see Piazza et al. 2001). As a consequence, $\mathrm{X}$-RAR $\alpha$ and RAR $\alpha$-X fusion genes are generated encoding aberrant fusion proteins that can interfere with RAR $\alpha$ and/or the X protein function (Fig. 2). Since the RAR $\alpha$ portion of the fusion protein is able to mediate heterodimerization with RXRs as well as DNA and ligand binding through the DNA and retinoic acid (RA) binding domains of RAR $\alpha$, respectively, X-RAR $\alpha$ fusion products always retain the ability to interfere with the RAR/RXR pathway at the transcription level. The various X-RAR $\alpha$ proteins also invariably display the

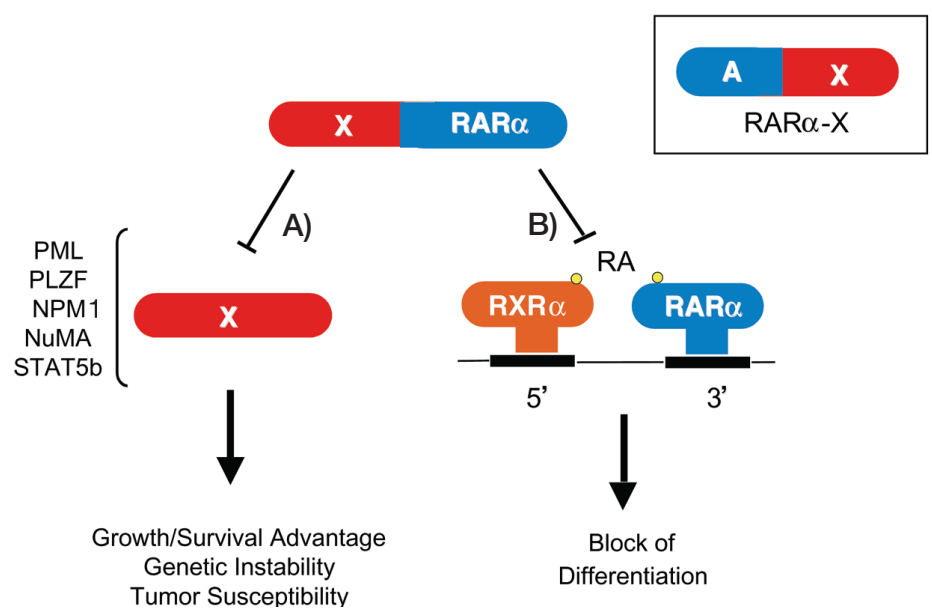

Figure 2. Fusion proteins in the pathogenesis of APL. APL is associated with specific chromosomal translocations where $R A R \alpha$ fuses with variable partner genes (X genes): PML, PLZF, NPM1, $N U M A$, and STAT5. As a consequence, X-RAR $\alpha$ and RAR $\alpha-X$ fusion genes are generated, which encode aberrant fusion proteins capable of interfering with $(A)$ the product of the remaining allele of the $\mathrm{X}$ gene (already reduced to heterozygosity as a consequence of the translocation), and $(B)$ the transcriptional activity of the $\operatorname{RAR} \alpha / \operatorname{RXR} \alpha$ heterodimeric complex. 
ability of heterodimerizing with the respective product of the remaining $X$ allele (e.g., PML-RAR $\alpha$ with PML, PLZF-RAR $\alpha$ with PLZF, etc.), suggesting that X-RAR $\alpha$ could simultaneously interfere with both $\mathrm{X}$ and RAR $\alpha$ pathways. Moreover, as a consequence of the translocation, the leukemia cell becomes heterozygous for both $\mathrm{X}$ and the RAR $\alpha$ genes. On the basis of these facts and-as soon as we cloned the PML gene- of preliminary observations that PML overexpression induced growth arrest and/or apoptosis depending on the cellular context, we originally proposed a working model by which the $\mathrm{X}$ genes could be regarded as leukemia suppressor genes whose function is interfered with by the APL fusion proteins. In vivo genetic testing of this hypothesis - initially studying PML and subsequently the other partners of RAR $\alpha$ - has on the one hand provided ample support to this notion, but on the other hand clearly revealed how the role of these APL genes in tumor suppression goes beyond the pathogenesis of this subtype of leukemia.

\section{PML Is a Pleiotropic Tumor Suppressor}

The ability of the APL-associated fusion products to interfere with the function of the $\mathrm{X}$ genes in a dominant negative fashion has been clearly demonstrated in the case of PML-RAR $\alpha$. PML is typically found in multiprotein speckled subnuclear structures termed PML nuclear bodies (PML-NBs) (Zhong et al. 2000a; Jensen et al. 2001; and references therein). In $\mathrm{Pml}^{-/-}$mouse primary cells, PML-NB components acquire an aberrant nuclear localization pattern that can be restored to normal when PML is added back (Zhong et al. 2000b). Thus, PML is essential for the formation and stability of PML-NB. With the use of antibodies against PML, it has been demonstrated that in the APL blasts PML-RAR $\alpha$ delocalizes PML into microspeckled nuclear structures through physical association, in turn causing the disruption of the PML-NBs (Melnick and Licht 1999; Zhong et al. 2000b). These observations lead then to the conclusion that PML may regulate the nuclear-body-associated functions of multiple nuclear body components (more than 50 proteins including $\mathrm{p} 53, \mathrm{Rb}$, Daxx, and $\mathrm{CBP}$ ), and that these functions may be impaired in the APL blast or in cells lacking PML function.

In fact, PML can modulate a number of tumor suppressive pathways (Salomoni and Pandolfi 2002). For instance, PML acts as a transcriptional coactivator of p53 and its family members p73 and p63 (Guo et al. 2000; Bernassola et al. 2004, 2005). It is also required for p53dependent induction of apoptosis and cellular senescence upon exposure to ionizing radiation and oncogenic transformation (Guo et al. 2000; Pearson et al. 2000). Moreover, $\mathrm{Pml}^{-1-}$ mice and cells are protected from multiple caspase-dependent pro-apoptotic stimuli, such as Fas, tumor necrosis factor, ceramide, and interferon treatment (Wang et al. 1998). More recently, we have shown that PML is also an essential modulator of transforming growth factor beta (TGF- $\beta$ ) signaling, a pluripotent cytokine that controls key tumor suppressive functions (Lin et al. 2004). Therefore, PML is implicated in the modulation of cellular functions such as apoptosis, growth sup- pression, and cellular senescence, that are essential for tumor suppression (Salomoni and Pandolfi 2002). On this basis, it was perhaps not surprising that $P m l$ inactivation in knockout (KO) mutants would induce a dramatic acceleration of the leukemic phenotype in mouse models of APL (Rego et al. 2001). However, PML-like many other leukemia/lymphoma-specific genes and the other genes of APL - is also dynamically expressed in non-hematopoietic cells. It was therefore tempting to hypothesize and test whether PML loss may participate in the pathogenesis of non-hematopoietic tumors as well. Indeed, using a highthroughput approach, we have recently demonstrated that PML expression is frequently lost in a number of epithelial tumor types such as, for instance, prostate cancer (Gurrieri et al. 2004). Importantly, PML loss correlated with tumor progression and grade (Gurrieri et al. 2004). The relevance of these findings is further corroborated by additional data obtained from $P m l$ null mice, which indeed demonstrated to be prone to spontaneously develop epithelial cancers (L.C. Trotman et al., in prep.). Taken together, this comprehensive analysis of the PML status in human cancers provides compelling evidence that leukemia genes may participate in the pathogenesis of malignancies other than APL, in this case through modulation of critical tumor suppressive pathways.

\section{THE NUCLEOPHOSMIN PARADIGM}

\section{Nucleophosmin Is Often Aberrant in Human Cancer}

Although a role for PML-RAR $\alpha$ as dominant negative over PML function is apparent and led to the discovery of PML as a pleiotropic tumor suppressor gene in human cancer, the same concept seemed at first difficult to apply to the NPM1 gene (another partner of RAR $\alpha$ in APL) and the correspondent fusion product NPM-RAR $\alpha$. In fact, nucleophosmin (NPM) has been traditionally regarded as a tumor marker and a putative proto-oncogene rather than a putative tumor suppressor gene, in view of the fact that its expression is typically up-regulated by mitogenic signals and correlates with cell proliferation and survival. Indeed, malignant and actively dividing cells express elevated levels of NPM, and cells expressing large amounts of NPM are resistant to apoptosis induced by either UV damage or hypoxia (Wu et al. 2002; Li et al. 2004). On the other hand, the NPM1 gene (genetic locus 5q35) is found translocated to other fusion partner genes beside RAR $\alpha$ (Redner et al. 1996) in a number of human hematopoietic malignancies: It rearranges with the $A L K$ gene in anaplastic large cell lymphomas (ALCL) (Morris et al. 1994) and with the $M L F 1$ gene in AML, CML, and myelodysplastic syndrome (MDS) (Yoneda-Kato et al. 1996). In addition, NPM has been recently found mutated and aberrantly localized in a high proportion of AML patients (Falini et al. 2005; Grisendi and Pandolfi 2005a). Although these observations strongly implicate NPM in the pathogenesis of human cancer, they make it difficult to visualize it as a putative oncogene or tumor suppressor gene exclusively. 


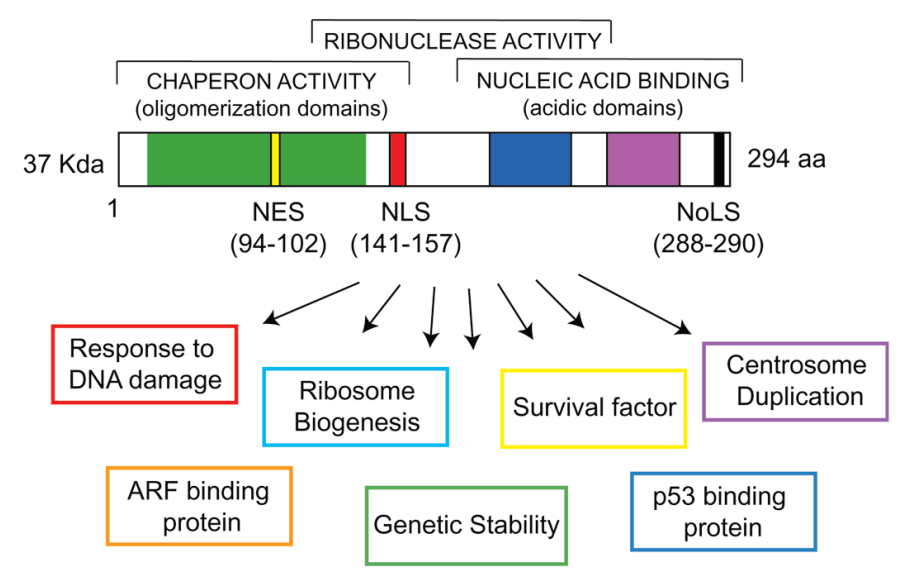

Figure 3. NPM is a multifunctional protein, composed of distinct functional domains which mediate diverse biochemical activities. In addition, NPM subcellular localization is tightly regulated by different localization signals: nuclear export signal (NES), nuclear localization signal (NLS), and nucleolar localization signal (NoLS). NPM participates in a number of biological processes: ribosome biogenesis, response to stress stimuli and DNA damage, control of centrosome duplication and genetic stability, and binding and stability control of key tumor suppressor genes such as p19 Arf and p53.

\section{The Complexity of NPM Biology}

As the involvement of NPM in human cancer is compelling but complex, the picture is further complicated by the multifaceted nature of this protein and its direct involvement in both proto-oncogenic and anti-oncogenic pathways. NPM is in fact a multifunctional protein (Fig. 3 ), which has been originally recognized as a nucleolar factor implicated in ribosome biogenesis and transport (Savkur and Olson 1998; Hingorani et al. 2000). Proliferating cells express higher NPM levels, and levels of expression of this protein seem to play an important role in protecting cells from apoptosis in different cell types (Pang et al. 2003; Ahn et al. 2005).

However, NPM also has been shown to associate to the centrosome and to inhibit its duplication in a cell-cycledependent manner controlled by phosphorylation by the CDK2-cyclin E complex (Okuda et al. 2000) and to be the target of mitotic kinases such as Nek2 and Plk1 (Yao et al. 2004; Zhang et al. 2004), involved in the control of proper cell division.

NPM has been proposed to modulate both positively and negatively the activity of major tumor suppressor genes such as $\mathrm{p} 53$ and p19 Arf. Although it seemingly stabilizes p53 upon overexpression in primary cells (Colombo et al. 2002), and protects 533 from HDM2-mediated degradation in response to UV irradiation (Kurki et al. 2004), NPM is also proposed to down-regulate p53 stability and antagonizes stress-induced apoptosis in human normal and malignant hematopoietic cells (Li et al. 2005). A comparable level of complexity applies to the recently discovered functional link between NPM and p19 $9^{\text {Arf }}$ tumor suppressor. Whereas $19^{\text {Arf }}$ has been described to induce NPM degradation and protein synthesis inhibition (Itahana et al. 2003) and to impede NPM shuttling in an Mdm2-sensitive pathway (Brady et al. 2004), NPM seems to play a role in both favoring $\mathrm{p} 19^{\text {Arf }}$ stability within high-molecular-weight protein complexes (Bertwistle et al. 2004) and inhibiting p19 ${ }^{\text {Arf }}$ cytoplasmic function by sequestering it inside the nucleolus (Korgaonkar et al. 2005). Thus, the literature is complex, and it is difficult to discern to which extent what is reported is the result of cell line- and overexpression-related artifacts.
In this laboratory, we have historically dissected the molecular genetics underlying APL and human cancer in the mouse through the use of a direct genetic approach in transgenic and $\mathrm{KO}$ models. Thus, in order to disentangle the complexity underlying the role of NPM in tumorigenesis, we generated not solely KO mutants, but rather a whole hypomorphic $\mathrm{Npm}$ mutant series $\left[\mathrm{Npm}^{+/-}<\right.$ $\left.\mathrm{Npm}^{\text {hy/hy }}<\mathrm{Npm}^{-1-}\right]$ in the mouse (Grisendi et al. 2005). To this end, we created a null allele by replacing 5 exons within the gene with a GFP coding sequence, as well as a hypomorphic allele by knocking the Neomycin cassette into an intronic region of the gene with the aim to interfere with the transcriptional activity of $\mathrm{Npm}$.

\section{A Role for Npm in the Regulation of the Centrosome Cycle}

The first important information we obtained from this approach is that total abrogation (null) or even a strong reduction (hypomorphic) in $\mathrm{Npm}$ expression results in embryonic lethality on average around embryonic day 11.5-16.5, depending on the residual expression levels of the gene. Detailed analysis of the mutant embryos revealed that $\mathrm{Npm}$ is an essential developmental regulator that exerts functions specifically required for proper forebrain and blood development (Grisendi et al. 2005). Collectively, these observations led to two surprising conclusions: First, although ultimately crucial for survival, Npm is unexpectedly dispensable for embryonic life up to midgestation, different from what is observed for other genes firmly involved in ribosome biogenesis. This suggests either that Npm is redundant in this function, at least up to mid-gestation, or that the extent of the defect in ribosome biogenesis caused by Npm inactivation is still compatible with cell proliferation. Second, Npm controls embryogenesis and organogenesis in a specific manner.

$\mathrm{Npm}^{-/}$embryos also showed high degrees of apoptosis. This was not totally unexpected since NPM has been described to be an important player in the apoptotic response and to protect cells from programmed cell death stimuli (see above). This increased apoptosis was accompanied by a robust $\mathrm{p} 53$ up-regulation, in spite of the fact that NPM has been described to act as a positive regulator of p53 stabilization upon overexpression (Colombo et 
al. 2002) and in response to stress stimuli (Kurki et al. 2004). This $\mathrm{p} 53$ response was also observed in embryonic fibroblasts (MEFs), where it takes place along with a concomitant up-regulation of $\mathrm{p} 21^{\mathrm{Waf} 1 / \mathrm{Cip} 1}$, resulting in a premature senescence phenotype. We therefore went in search of a possible explanation for this p53 response. DNA damage and genomic instability are known to trigger a p53 response (Levine 1997). An increase in apoptosis accompanied by p53 up-regulation is observed in mutants such as Brca1-deficient embryos, whose cells suffer from genetic instability (Xu et al. 1999, 2001). Interestingly, we observed that $\mathrm{Npm}$-deficient cells consistently underwent cell cycle arrest and exhibit a 4N DNA content. Polyploidy was detectable by cell cycle and DNA content analysis, suggesting that Npm loss or hypomorphism triggered a p53-dependent postmitotic tetraploid cell cycle arrest, as previously described in MEFs subjected to mitotic spindle disruption (Lanni and Jacks 1998). This reasoning is supported by the aforementioned increased expression of $\mathrm{p} 21^{\mathrm{Waf1} / \mathrm{Cip} 1}$, typically known to mediate cell cycle arrest in $\mathrm{G}_{1}$ phase, and lack of up-regulation of $\mathrm{G}_{2}-\mathrm{M}$ markers (e.g., phospho-histone $\mathrm{H} 3$ and cyclin B). We therefore investigated the status of mitotic spindles in $\mathrm{Npm}^{--}$cells and, to our surprise, we found significant numbers of aberrant mitotic figures characterized by the presence of multipolar spindles as well as multiple functional centrosomes. $\mathrm{Npm}^{-/-}$interphase MEFs display centrosome amplification (3 or more centrosomes per cell) in up to $30 \%$ of the cell population. Furthermore, blood precursor cells isolated from the yolk sac of $\mathrm{Npm}^{-1-}$ mutant embryos also show a massive increase in the number of centrosomes detectable in large multinucleated cells with up to 4 nuclei. Taken together, these findings suggest that Npm loss of function may lead to inappropriate centrosome duplication (Fig. 4). This in turn may cause centrosome amplification accompanied by aberrant cell division, and these effects ultimately contribute to the developmental defects observed in the Npm mutant embryos.

\section{Npm Can Act as a Bona Fide Tumor Suppressor}

Although less drastically affected, early-passage $\mathrm{Npm}^{+/-}$also displayed decreased cell proliferation rates and supernumerary centrosomes. Moreover, chromosome FISH analysis revealed significant levels of tetraploidy and aneuploidy not only in Npm null and hypomorphic cells, but also in heterozygous MEFs in a dose-dependent manner. Because the NPM1 gene dosage is reduced by half in cancer cells harboring chromosomal rearrangements/deletions at the NPM1 locus, we hypothesized that Npm could be haploinsufficient in the control of centrosome duplication and genomic stability and, hence, behave as a haploinsufficient tumor suppressor. We tested this possibility both in vitro using once again MEFs, and in vivo. In contrast to $\mathrm{Npm}^{-/-}$and hypomorphic MEFs, which eventually stop proliferating and undergo irreversible senescence, $\mathrm{Npm}^{+/}$and wild-type cells could be maintained in culture for a comparable number of passages. This is likely due to the fact that in $\mathrm{Npm}^{+/-}$
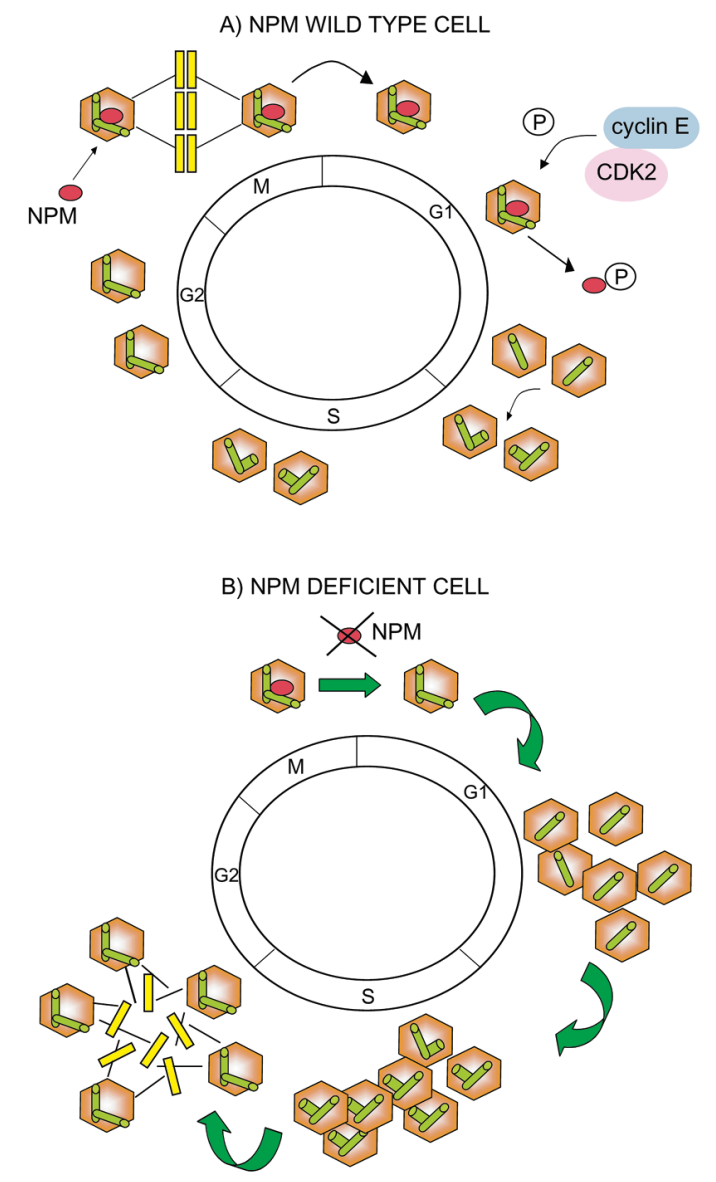

Figure 4. NPM loss of function causes centrosome amplification. (A) In the normal condition, NPM localizes to the centrosome during mitosis and the early $\mathrm{G}_{1}$ phase of the cell's cycle. During the $\mathrm{G}_{1}-\mathrm{S}$ phase transition, NPM is phosphorylated by the CDK2-cyclin E complex and dissociates from the centrosome. This allows centriole separation, and the centrosome duplicates. NPM is kept phosphorylated and separated from the centrosome until the end of the $\mathrm{G}_{2}$ phase, when it re-associates to the centrosome at mitosis. $(B)$ A reduction in NPM expression results in untimely and/or unrestricted centrosome duplication, leading to the formation of additional centrosomes and multipolar spindles. Aberrant mitosis in turn results in unbalanced chromosome segregation and, eventually, aneuploidy.

cells the defects observed are milder, still allowing the cells to survive and proliferate, although at a different rate. We therefore used $\mathrm{Npm}^{+/-}$cells to investigate whether the observed genetic instability arising from centrosome defects would affect the ability of these cells to immortalize in culture and their susceptibility toward cellular immortalization and transformation. In a stringent 3T9 protocol, at early passages, the proliferation rates of $\mathrm{Npm}^{+/-}$MEFs were lower, but, upon immortalization, the same cells acquired considerably higher proliferation rates compared to wild-type controls. This was accompanied by higher aneuploidy and a greater ability to efficiently form transformation foci in low-density seeding assays. In addition, upon immortalization, $\mathrm{Npm}^{+/-} \mathrm{MEFs}$ also display a higher susceptibility to transformation induced by acute expression of known oncogenes, such as 
$\operatorname{Ras}^{\mathrm{V} 12}$ or c-myc. Taken together, these observations suggest that the Npm gene dosage is a critical determinant in maintaining proper centrosome numbers and chromosomal stability, and that the underlying genetic instability exhibited by $\mathrm{Npm}$-deficient cells is a driving force toward faster acquisition of additional genetic mutations required initially for immortalization and then for transformation.

This working model is further supported by what we observed in vivo in $\mathrm{Npm}^{+/-} / \mathrm{E} \mu$-myc transgenic compound mice, which we generated in order to determine whether Npm heterozygous inactivation increases susceptibility to lymphomagenesis. Not only was the onset of lymphoma in $\mathrm{Npm}^{+-}$; E $\mu$-myc compound mice significantly accelerated in comparison with the E $\mu$-myc cohort, but, importantly, the karyology of these lymphomas was profoundly affected by the heterozygous loss of $\mathrm{Npm}$. Whereas tumors from $\mathrm{Npm}^{+-}$; E $\mathrm{\mu}$-myc mice displayed mostly numerical abnormalities, in contrast, in tumors from the E $\mu$-myc cohort we observed mostly structural abnormalities, as previously reported.

Npm plays a role in hematopoiesis during embryonic development as hematopoietic precursors in the yolk sac are drastically reduced in number and differentiation ability. Since Npm proved to be haploinsufficient in the control of cellular ploidy, we asked whether this would translate into an overt phenotype in the hematopoiesis of $\mathrm{Npm}^{+/-}$mutants. Remarkably, $\mathrm{Npm}^{+/-}$mice displayed features resembling some of those identified in the human MDS, such as, for instance, signs of dyserythropoiesis in the bone marrow, with a high proportion of dysplastic and bi-nucleated erythroid cells, as well as hypercellularity of red blood cell precursors. These observations are particularly relevant in view of the fact that deletion of variable portions of the long arm of chromosome 5 (5q) or of the whole chromosome 5 is frequently observed in patients with de novo and therapy-related MDS (for review, see Olney and Le Beau 2002). The deleted fragment (often located between bands $5 q 13$ and 5q35) is variable in size and breakpoint localization, and it can be either the sole genetic anomaly or it can be accompanied by additional structural and numerical chromosomal changes. MDS is therefore regarded as a genetically and clinically heterogeneous condition where more than one gene may contribute to the final pathological outcome, although to date no gene has been firmly linked to the pathogenesis of these syndromes. Our findings indicate NPMI heterozygous loss as one of the genetic events that, in combination with loss of other genes located in the deleted region, could cooperate in the pathogenesis of full-blown human MDS.

Importantly, loss of $5 \mathrm{q} 35$, the genetic region where NPM1 resides, is frequently observed in non-hematopoietic tumors. For instance, it is a frequent event in nonsmall-cell lung carcinoma (Mendes-da-Silva et al. 2000). Loss of $5 \mathrm{q}$ and consequent NPM haploinsufficiency could therefore constitute an engine toward genomic instability in these tumors, in turn favoring tumor progression. Taken together, these observations once again indicate that the role of NPM in tumorigenesis might not be solely confined to hematopoietic malignancies.

\section{Oncogene or Tumor Suppressor: Is the Answer in the Dose?}

We found that Npm is required to maintain genomic integrity through the regulation of centrosome duplication and cellular ploidy. NPM is haploinsufficient for this function since $\mathrm{Npm}$ heterozygous cells display a significant degree of genomic instability, which results in increased susceptibility to oncogenic transformation both in vitro and in vivo. The acquisition of genetic mutations is a crucial driving force during tumorigenesis, in both solid and hematopoietic malignancies. The importance of this aspect is underlined by the fact that the maintenance of genetic stability is a tightly regulated biological function, and that crucial and pleiotropic tumor suppressor genes are strictly involved in the growth arrest/apoptotic response to DNA damage insults and aneuploidy (e.g., $\mathrm{p} 53)$. Alteration in NPM function might therefore have a larger impact on tumorigenesis, not only restricted to the field of hematopoietic disorders. This is especially relevant in view of the fact that not only is NPM translocated in a number of leukemia/lymphomas - determining a heterozygous state - and deleted in a proportion of cases of MDS, but it is also frequently lost in solid tumors (Mendes-da-Silva et al. 2000). However, NPM is also found overexpressed in a number of human cancers (both solid tumors and hematopoietic disorders). Thus, NPM could fall in a novel category of genes that act both as oncogene and tumor suppressor gene, depending on expression levels and gene dosage. Either a partial functional loss or an aberrant overexpression of this type of cancer gene would lead to neoplastic transformation through distinct mechanisms (Fig. 5).

\section{CONCLUSIONS}

We have discussed here representative examples in support of a more global role for "leukemia/lymphoma" genes in the pathogenesis of human cancer. Many of these genes in fact participate in the control of fundamental oncogenic and tumor suppressive networks that are shared by several cell types rather than being unique to hematopoietic malignancies. Even though a deregulated function of leukemia- or lymphoma-related genes may have differential outcomes and varying degrees of penetrance depending on the tissue/cell context, a systematic analysis of their status in human cancer is warranted. It would be wise to take advantage of the dramatic progress made in the last 20 years in determining the molecular genetics of most hematological malignancies and to apply this detailed knowledge to the analysis of other tumors whose genetic bases are still poorly understood. Thus, if we are to embark on a cancer genome anatomy project enterprise, we ought to pay particular attention to this specific subset of genes already directly implicated in the pathogenesis of human cancer. More in general, it could be very insightful to determine in detail the status of these genes in any given tumor type, address their function (if any) in the corresponding normal cell/type, and relate these functions to known oncogenic and tumor suppressive pathways in a systematic fashion. 


\section{GRISENDI AND PANDOLFI}

A) NPM OVEREXPRESSION
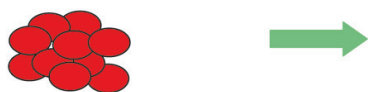

TRANSFORMATION:

-increased proliferation

-protection from apoptosis

B) NPM HETEROZYGOSITY (translocation or deletion)
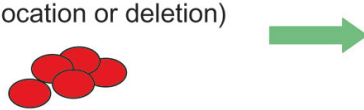

GENETIC INSTABILITY \& SUSCEPTIBILITY TO TRANSFORMATION:

-centrosome amplification

C) NPM COMPLETE LOSS

(conditional targeting)

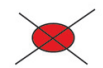

\section{CELL CYCLE ARREST:}

-post-mitotic growth arrest

\section{TUMORIGENESIS?}

Figure 5. The dose of NPM in tumorigenesis. The dose of the NPM gene dictates distinct outcomes in tumorigenesis: Whereas NPM overexpression may promote the growth of cancerous cells due to increased cell proliferation and inhibition of the apoptotic response $(A)$, NPM heterozygosity (or further dose reduction of NPM below $50 \%$ ) renders the cells genetically unstable and susceptible to transformation $(B)$. As the complete loss in NPM expression induces cell cycle arrest and cellular senescence $(C)$, it remains to be determined whether complete somatic loss of the NPM gene would be compatible and selected for by the transformed cell.

\section{ACKNOWLEDGMENTS}

We thank all past and present members of the Pandolfi laboratory, and Linda DiSantis for editing of the manuscript.

\section{REFERENCES}

Adams J.M., Harris A.W., Pinkert C.A., Corcoran L.M., Alexander W.S., Cory S., Palmiter R.D., and Brinster R.L. 1985. The c-myc oncogene driven by immunoglobulin enhancers induces lymphoid malignancy in transgenic mice. Nature 318: 533.

Ahn J.Y., Liu X., Cheng D., Peng J., Chan P.K., Wade P.A., and Ye K. 2005. Nucleophosmin/B23, a nuclear PI $(3,4,5) P(3)$ receptor, mediates the anti-apoptotic actions of NGF by inhibiting CAD. Mol. Cell 18: 435.

Arvanitis C. and Felsher D.W. 2005. Conditionally MYC: Insights from novel transgenic models. Cancer Lett. 226: 95.

Bernassola F., Oberst A., Melino G., and Pandolfi P.P. 2005. The promyelocytic leukaemia protein tumour suppressor functions as a transcriptional regulator of p63. Oncogene 24: 6982.

Bernassola F., Salomoni P., Oberst A., Di Como C.J., Pagano M., Melino G., and Pandolfi P.P. 2004. Ubiquitin-dependent degradation of p73 is inhibited by PML. J. Exp. Med. 199: 1545.

Bertwistle D., Sugimoto M., and Sherr C.J. 2004. Physical and functional interactions of the Arf tumor suppressor protein with nucleophosmin/B23. Mol. Cell. Biol. 24: 985.

Brady S.N., Yu Y., Maggi L.B., Jr., and Weber J.D. 2004. ARF impedes NPM/B23 shuttling in an Mdm2-sensitive tumor suppressor pathway. Mol. Cell. Biol. 24: 9327.

Colombo E., Marine J.C., Danovi D., Falini B., and Pelicci P.G. 2002. Nucleophosmin regulates the stability and transcriptional activity of p53. Nat. Cell Biol. 4: 529.

Dalla-Favera R., Bregni M., Erikson J., Patterson D., Gallo R.C., and Croce C.M. 1982. Human c-myc oncogene is located on the region of chromosome 8 that is translocated in Burkitt lymphoma cells. Proc. Natl. Acad. Sci. 79: 7824.

Falini B., Mecucci C., Tiacci E., Alcalay M., Rosati R., Pasqualucci L., La Starza R., Diverio D., Colombo E., Santucci A., Bigerna B., Pacini R., Pucciarini A., Liso A., Vignetti M., Fazi P., Meani N., Pettirossi V., Saglio G., Mandelli
F., Lo-Coco F., Pelicci P.G., and Martelli M.F. 2005. Cytoplasmic nucleophosmin in acute myelogenous leukemia with a normal karyotype. N. Engl. J. Med. 352: 254.

Grisendi S. and Pandolfi P.P. 2005. NPM mutations in acute myelogenous leukemia. N. Engl. J. Med. 352: 291.

Grisendi S., Bernardi R., Rossi M., Cheng K., Khandker L., Manova K., and Pandolfi P.P. 2005. Role of nucleophosmin in embryonic development and tumorigenesis. Nature 437: 147.

Guo A., Salomoni P., Luo J., Shih A., Zhong S., Gu W., and Pandolfi P.P. 2000. The function of PML in p53-dependent apoptosis. Nat. Cell Biol. 2: 730

Gurrieri C., Capodieci P., Bernardi R., Scaglioni P.P., Nafa K., Rush L.J., Verbel D.A., Cordon-Cardo C., and Pandolfi P.P. 2004. Loss of the tumor suppressor PML in human cancers of multiple histologic origins. J. Natl. Cancer Inst. 96: 269.

Halmos B., Huettner C.S., Kocher O., Ferenczi K., Karp D.D., and Tenen D.G. 2002. Down-regulation and antiproliferative role of C/EBPalpha in lung cancer. Cancer Res. 62: 528.

Hingorani K., Szebeni A., and Olson M.O. 2000. Mapping the functional domains of nucleolar protein B23. J. Biol. Chem. 275: 24451.

Itahana K., Bhat K.P., Jin A., Itahana Y., Hawke D., Kobayashi R., and Zhang Y. 2003. Tumor suppressor ARF degrades B23, a nucleolar protein involved in ribosome biogenesis and cell proliferation. Mol. Cell 12: 1151.

Jensen K., Shiels C., and Freemont P.S. 2001. PML protein isoforms and the RBCC/TRIM motif. Oncogene 20: 7223.

Korgaonkar C., Hagen J., Tompkins V., Frazier A.A., Allamargot C., Quelle F.W., and Quelle D.E. 2005. Nucleophosmin (B23) targets ARF to nucleoli and inhibits its function. Mol. Cell. Biol. 25: 1258 .

Kurki S., Peltonen K., Latonen L., Kiviharju T.M., Ojala P.M., Meek D., and Laiho M. 2004. Nucleolar protein NPM interacts with HDM2 and protects tumor suppressor protein p53 from HDM2-mediated degradation. Cancer Cell 5: 465.

Lanni J.S. and Jacks T. 1998. Characterization of the p53-dependent postmitotic checkpoint following spindle disruption. Mol. Cell. Biol. 18: 1055.

Levine A.J. 1997. p53, the cellular gatekeeper for growth and division. Cell 88: 323

Li J., Zhang X., Sejas D.P., and Pang Q. 2005. Negative regulation of $\mathrm{p} 53$ by nucleophosmin antagonizes stress-induced apoptosis in human normal and malignant hematopoietic cells. Leuk. Res. 29: 1415. 
Li J., Zhang X., Sejas D.P., Bagby G.C., and Pang Q. 2004. Hypoxia-induced nucleophosmin protects cell death through inhibition of p53. J. Biol. Chem. 279: 41275.

Li Q.L., Ito K., Sakakura C., Fukamachi H., Inoue K., Chi X.Z., Lee K.Y., Nomura S., Lee C.W., Han S.B., Kim H.M., Kim W.J., Yamamoto H., Yamashita N., Yano T., Ikeda T., Itohara S., Inazawa J., Abe T., Hagiwara A., Yamagishi H., Ooe A., Kaneda A., Sugimura T., Ushijima T., Bae S.C., and Ito Y. 2002. Causal relationship between the loss of RUNX3 expression and gastric cancer. Cell 5: 113 .

Lin H.K., Bergmann S., and Pandolfi P.P. 2004. Cytoplasmic PML function in TGF-beta signalling. Nature 431: 205.

Melnick A. and Licht J.D. 1999. Deconstructing a disease: RAR $\alpha$, its fusion partners, and their roles in the pathogenesis of acute promyelocytic leukemia. Blood 93: 3167.

Mendes-da-Silva P., Moreira A., Duro-da-Costa J., Matias D. and Monteiro C. 2000. Frequent loss of heterozygosity on chromosome 5 in non-small cell lung carcinoma. Mol. Pathol. 53: 184

Morgenbesser S.D. and DePinho R.A. 1994. Use of transgenic mice to study myc family gene function in normal mammalian development and in cancer. Semin. Cancer Biol. 5: 21.

Morris S.W., Kirstein M.N., Valentine M.B., Dittmer K.G., Shapiro D.N., Saltman D.L., and Look A.T. 1994. Fusion of a kinase gene, ALK, to a nucleolar protein gene, NPM, in nonHodgkin's lymphoma. Science 263: 1281.

Nesbit C.E., Tersak J.M., and Prochownik E.V. 1999. MYC oncogenes and human neoplastic disease. Oncogene 18: 3004.

Okuda M., Horn H.F., Tarapore P., Tokuyama Y., Smulian A.G., Chan P.K., Knudsen E.S., Hofmann I.A., Snyder J.D., Bove K.E., and Fukasawa K. 2000. Nucleophosmin/B23 is a target of CDK2/cyclin E in centrosome duplication. Cell 103: 127.

Olney H.J. and Le Beau M.M. 2002. The cytogenetics and molecular biology of the myelodysplastic syndromes. In The myelodysplastic syndromes, pathobiology and clinical management (ed. J.M. Bennet), p. 89. Marcel Dekker, New York.

Oster S.K., Ho C.S.W., Soucie E.L., and Penn L.Z. 2002. The myc oncogene: Marvelously complex. Adv. Cancer Res. 84: 81

Pang Q., Christianson T.A., Koretsky T., Carlson H., David L., Keeble W., Faulkner G.R., Speckhart A., and Bagby G.C. 2003. Nucleophosmin interacts with and inhibits the catalytic function of eukaryotic initiation factor 2 kinase PKR. J. Biol. Chem. 278: 417091.

Pearson M., Carbone R., Sebastiani C., Cioce M., Fagioli M, Saito S., Higashimoto Y., Appella E., Minucci S., Pandolfi P.P., and Pelicci P.G. 2000. PML regulates p53 acetylation and premature senescence induced by oncogenic Ras. Nature 406: 207.

Piazza F., Gurrieri C., and Pandolfi P.P. 2001. The theory of APL. Oncogene 20: 7216.

Redner R.L., Rush E.A., Faas S., Rudert W.A., and Corey S. J. 1996. The $\mathrm{t}(5 ; 17)$ variant of acute promyelocytic leukemia expresses a nucleophosmin-retinoic acid receptor fusion. Blood 87: 882.
Rego E.M., Wang Z.G., Peruzzi D., He L.Z., Cordon-Cardo C., and Pandolfi P.P. 2001. Role of promyelocytic leukemia (PML) protein in tumor suppression. J. Exp. Med. 193: 521.

Salomoni P. and Pandolfi P.P. 2002. The role of PML in tumor suppression. Cell 108: 165.

Savkur R.S. and Olson M.O. 1998. Preferential cleavage in preribosomal RNA by protein B23 endoribonuclease. Nucleic Acids Res. 26: 4508.

Spencer C.A. and Groudine M. 1991. Control of c-myc regulation in normal and neoplastic cells. Adv. Cancer Res. 56: 1.

Tomlins S.A., Rhodes D.R., Perner S., Dhanasekaran S.M., Mehra R., Sun X., Varambally S., Cao X., Tchinda J., Kuefer R., Lee C., Montie J.E., Shah R.B., Pienta K.J., Rubin M.A., and Chinnaiyan A.M. 2005. Recurrent fusion of TMPRSS2 and ETS transcription factor genes in prostate cancer. Science 310: 644.

Vennstrom B., Sheiness D., Zabielski J., and Bishop J.M. 1982. Isolation and characterization of c-myc, a cellular homolog of the oncogene (v-myc) of avian myelocytomatosis virus strain 29. J. Virol. 42: 773.

Wang Z.G., Ruggero D., Ronchetti S., Zhong S., Gaboli M., Rivi R., and Pandolfi P.P. 1998. PML is essential for multiple apoptotic pathways. Nat. Genet. 20: 266.

Wu M.H., Chang J.H., and Yung B.Y.M. 2002. Resistance to UV-induced cell-killing in nucleophosmin/B23 over-expressed NIH3T3 fibroblasts: Enhancement of DNA repair and up-regulation of PCNA in association with nucleophosmin/B23 over-expression. Carcinogenesis 23: 93.

Xu X., Qiao W., Linke S.P., Cao L., Li W.M., Furth P.A., Harris C.C., and Deng C.X. 2001. Genetic interactions between tumor suppressor Brca1 and p53 in apoptosis, cell cycle and tumorigenesis. Nat. Genet. 28: 266.

Xu X., Weaver Z., Linke S.P., Li C., Gotay J., Wang X.W., Harris C.C., Ried T., and Deng C.X. 1999. Centrosome amplification and defective G2-M cell cycle check-point induce genetic instability in BRCA1 exon 11 isoform-deficient cells. Mol. Cell 3: 389.

Yao J., Fu C., Ding X., Guo Z., Zenreski A., Chen Y., Ahmed K., Liao J., Dou Z., and Yao X. 2004. Nek2A kinase regulates the localization of numatrin to centrosome in mitosis. FEBS Lett. 575: 112 .

Yoneda-Kato N., Look A.T., Kirstein M.N., Valentine M.B., Raimondi S.C., Cohen K.J., Carroll A.J., and Morris S.W. 1996. The $\mathrm{t}(3 ; 5)(\mathrm{q} 25.1 ; \mathrm{q} 34)$ of myelodysplastic syndrome and acute myeloid leukemia produces a novel fusion gene, NPM-MLF1. Oncogene 12: 265.

Zhang H., Shi X., Paddon H., Hampong M., Dai W., and Pelech S. 2004. B23/nucleophosmin serine 4 phosphorylation mediates mitotic functions of polo-like kinase 1. J. Biol. Chem. 279: 35726 .

Zhong S., Salomoni P. , and Pandolfi P.P. 2000a. The transcriptional role of PML and the nuclear body. Nat. Cell Biol. 2: E85.

Zhong S., Muller S., Ronchetti S., Freemont P.S., Dejean A., and Pandolfi P.P. 2000b. Role of SUMO-1 modified PML in nuclear body formation. Blood 95: 2748. 


\title{
$\$_{\text {SSH\& }}^{\infty} \mathrm{CS}$ Cold Spring Harbor Symposia SYMPOSIA on Quantitative Biology
}

\section{Two Decades of Cancer Genetics: From Specificity to Pleiotropic Networks}

\author{
S. GRISENDI and P.P. PANDOLFI
}

Cold Spring Harb Symp Quant Biol 2005 70: 83-91

Access the most recent version at doi:10.1101/sqb.2005.70.023

References This article cites 52 articles, 18 of which can be accessed free at: http://symposium.cshlp.org/content/70/83.full.html\#ref-list-1

License

Email Alerting Receive free email alerts when new articles cite this article - sign up in Service the box at the top right corner of the article or click here. 